\title{
Barchan Sand Dunes Collisions Detection in High Resolution Satellite Images based on Image Clustering and Transfer Learning
}

\author{
M. A. Azzaoui ${ }^{1}$, L. Masmoudi ${ }^{2}$ \\ Laboratoire Conception et Systèmes: \\ Électronique, signaux et informatique \\ Université Mohammed V de Rabat \\ Morocco
}

\author{
H. El Belrhiti ${ }^{3}$ \\ Département des Sciences \\ Fondamentales et Appliquées \\ Institut Agronomique et Vétérinaire \\ Hassan II, Rabat, Morocco
}

\author{
I. E. Chaouki ${ }^{4}$ \\ Département Informatique \\ Ecole Nationale des Sciences \\ Appliquées d'Agadir \\ Agadir, Morocco
}

\begin{abstract}
Desertification is a core concern for populations living in arid and semi-arid areas. Specifically, barchans dunes which are the fastest moving sand dunes put constant pressure over human settlements and infrastructure. Remote sensing was used to analyze sand dunes around Tarfaya city located in the south of Morocco in the Sahara Desert. In this area, dunes form long corridors made of thousands crescent shaped dunes moving simultaneously, thus, making data gathering in the field very difficult. A computer vision approach based on machine learning was proposed to automate the detection of barchans sand dunes and monitor their complex interactions. An IKONOS high resolution satellite image was used with the combination of a clustering algorithm for image segmentation of the dunes corridor, and a Transfer Learning model which was trained to detect three classes of objects: Barchan dunes, bare fields and a new introduced class consisting of dunes collisions. Indeed, collisions were very difficult to model using classical digital image processing methods due to the large variability of their shapes. The model was trained on 1000 image patches which were annotated then augmented to generate a larger dataset. The obtained detection results showed an accuracy of $84,01 \%$. The interest of this research was to provide with a relatively affordable approach for tracking sand dunes locations in order to better understand their dynamics.
\end{abstract}

Keywords - High resolution satellite images; remote sensing; transfer learning; image segmentation; sand dunes; desertification

\section{INTRODUCTION}

\section{A. Barchan Dunes and Desertification Issue}

The United Nations declared desertification as a phenomenon affecting 250 million people worldwide and increasing in intensity as a result of climate change [1]. Not all dunes contribute equally to the desertification problem. Indeed, the taxonomy of sand dunes includes multiple dune categories, posing different risk levels depending on their velocities [2]. The 3 main categories of sand dunes are: Star dunes, Linear dunes and Barchan dunes. The first ones called Star Dunes form in areas where winds blow from multiple directions, thus limiting their moving capabilities. The second ones called Linear Dunes form under bi-directional winds that define their longitudinal shape, thus making them relatively slow. The third ones called Barchans form under unidirectional winds, thus, they are the fastest moving sand dunes, and have characteristic crescent shape [3]. In the context of natural hazard monitoring, data gathering is compulsory to anticipate barchans dunes risk. However, it is obviously expensive and difficult to gather field data regularly at such a large scale in a harsh environment. Hence, the use of remote sensing, which was a convenient solution.

\section{B. Problem Statement}

Remote sensing is essential for monitoring sand dunes, however, as deserts contain thousands of dunes; and their annotation is executed manually, this task becomes time consuming. Therefore, the proposed approach consisted of investigating the use of computer vision algorithms to automate barchans dunes detection and segmentation. Barchan dunes had a characteristic crescent shape, which could be segmented using classical computer vision algorithms [4]. However, in the field, thousands of barchans moved simultaneously, with smaller dunes moving faster than larger ones, resulting into dune collisions. This further complicates the problem, as dunes collisions had odd shapes which require more advanced computer vision algorithms to be detected. Therefore, in this study, Transfer Learning was introduced to detect barchans dunes collisions.

\section{Aim of Research}

The proposed research study allowed to gather data through the use of high resolution satellite imagery along with a machine learning approach. The goal was to provide with valuable information, not only to geologists, which would to improve their understanding of desertification phenomenon, but also, to urban planners towards the selection of sustainable solutions for reducing the effect of moving sand dunes on the daily lives of the local inhabitants and all stakeholders, and also for decision makers, to better anticipate the risks of moving sand dunes and limit desertification phenomenon impact on the populations.

\section{Sand Dunes Detection using Remote Sensing}

Sand dunes detection is an example of application for which the use remote sensing was essential. In fact, scientists started using it early on for the categorization of sand dunes: [5] used Landsat (ERTS) mosaics to create a global map of sand 'seas'. [6] used QuickBird satellite images to observe 
morphometric changes in sand dunes. Aerial photogrammetry was also common: [7] analysed the self-organizing patterns of sand dunes. [8] used Google maps images to confirm simulations for dunes stabilization. However, dunes mapping was mostly done manually. With the development of digital image processing, the use of satellite images became more efficient and more information could be extracted from images. During this phase, pixel based approaches were preponderant in the literature [9], [10], [11], and spatial and spectral properties of sand dunes were extensively exploited to gather morphology measurements and study their dynamics, based on ASTER, IKONOS or Landsat satellite images. Later on, with the development of computer vision, researchers in remote sensing shifted to Geospatial Object Based Image Analysis (GEOBIA) paradigm, and dunes detection approaches were based on objects characteristics such as shape, motion, colour and texture [12]. With the development of image descriptors such as (GLCM) Gray-Level Co-occurrence Matrix for texture, Histogram of Oriented Gradients (HOG), Local Binary Patterns (LBP) and Speed-Up Robust Features (SURF), more approaches were focused on feature engineering, and the use of classifiers with ensemble classifiers such as random forest (RF), and as support vector machine (SVM) to achieve dunes detections results. In recent years, the advances made in machine learning and especially Neural Networks with Deep Learning became more popular in remote sensing.

\section{E. Machine Learning and Transfer Learning for Satellite Remote Sensing}

Machine Learning includes many training algorithms and models, among which Support Vector Machines (SVM) and Multi-Layer Perceptrons (MLP) which could be used to solve non-linear problems. Indeed, in the case of (MLP), the model is composed by a set of hidden layers which learn successively higher level features, in addition to an input layer and an output layer rendering the classification [13]. Layers were made of neurons which were characterized by weight coefficients $w_{i}$, and an activation function $\phi$. When the number of hidden layers is important, the model gets called Deep Learning [14]. Deep Learning was also trained using Backpropagation algorithm which dates back to the 90s. During the 2000s, other machine learning algorithms and models such as (SVM) were outperforming (MLPs), and adding layers was considered as an explosion of free parameters, producing a high VC dimension, which would result into overfitting. The turning point came on 2012 when AlexNet, a Convolutional Neural Network (CNN) won the ImageNet competition by a significant margin, by combining the use of a large dataset, rectified linear units (ReLUs) as activation functions, and graphics processing units (GPUs). Computer vision community gained interest and other classes of Deep Learning models were re-explored and developed: Autoencoders (AE) which were used for unsupervised learning, Deep Belief Networks (DBN), and also Generative Adversarial Networks (GAN) models, where two neural networks competed with each other as in game theory [15]. Other developments took place in the recent years with VGG Networks [16], RESNET [17] and Inception-v4 [18].

Remote sensing researchers have used Deep Learning in different applications: [19] for hyperspectral data classification, [20] reviewed Deep Learning for in Land Use and Land Cover with applications related to urbanization. [21] showed the use of Deep Learning for image processing, object detection and classification, including aircraft segmentation, vehicle detection, and scene identification. However, to the extent of our knowledge, no study applied Deep Learning and its variant called Transfer Learning which will be presented in detail in the next section, for the detection of Barchan dunes and sand dunes collisions in high resolution satellite images.

\section{F. Our Contribution}

The former paragraphs exposed the importance of barchan dunes detection for treating the desertification issue. They also showed how dunes mapping required remote sensing, and explained that the current approaches for mapping sand dunes were manual and occasionally, made use of basic digital image processing, which limited their capabilities to objects with well-defined shapes. This is where this contribution intervenes: As existing approaches could not generalize well to the variability of dunes shapes and especially sand dunes collisions, this paper introduced a new approach that combined Transfer Learning which is based on Deep Learning, and image clustering for the detection of Barchan dunes and their collisions in remote sensing satellite images. In the next sections, the framework used will be presented in details, along with the obtained results, a discussion of future works, and the conclusion.

\section{PROPOSED WORK}

The proposed method was composed of three major steps: First, it began with a pre-processing step on the satellite image, in order to increase the spatial resolution of multispectral bands, reduce noise and enhance image contrast. Second, it used an image clustering method to segment sand regions and distinguish them from the bedrock. Third, it trained a Transfer Learning framework on areas which were segmented previously, thus combining both methods to predict three classes of objects in the satellite image: barchans dunes, dunes collisions and bare field. In the following, first, a brief background presentation is presented for Transfer Learning, which is a derivation of a Deep Learning model. Next, the different steps of the proposed method are explained in detail.

\section{A. Transfer / Deep Learning Background}

Transfer Learning is an approach used to bootstrap the learning of a new task, by transferring knowledge from a similar task already learned, and adapting it. In our case, it was Deep Learning AlexNet architecture. AlexNet is a Deep Learning Convolutional Neural Network (CNN) which was first introduced during ImageNet contest where it outperformed state of art methods by $10 \%$ [22]. Its structure consisted of 8 layers: 3 connected layers and 5 convolutional layers. illustrates its architecture which was composed of 60 million parameters and 650,000 neurons in total. This model took 3 channels RGB images of size $227 \times 227$, which went through its consecutive layers (Fig.1).

The first layer was a convolutional layer with 96 kernels having a size of $11 \times 11 \times 3$ and a ReLU non-linear activation function (1).

$\emptyset(x)=\max (0, x)$ 


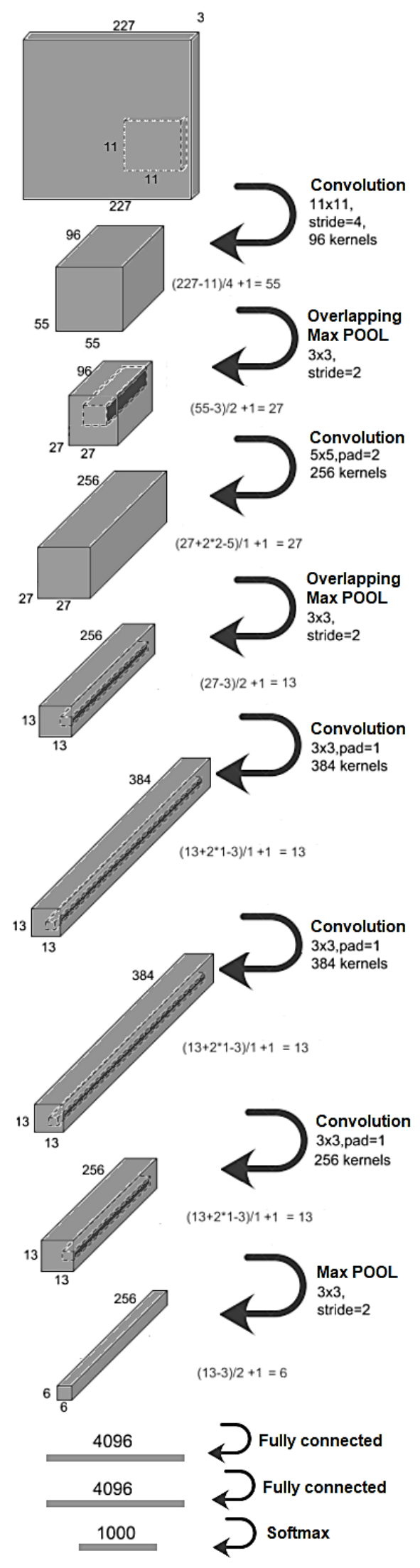

Fig. 1. Deep Learning Architecture of AlexNet.
With ? the weighted neuron input. The second layer was an Overlapping Max Pooling layer which conserved the depth, while down sampling the width and height of the tensors. The stride of 2 is used as an overlapping between adjacent windows in order to reduce the error rate. The following layer in another convolutional layer, followed by another overlapping max pooling layer, then three convolutional layers, then a max pooling layer, followed by two fully connected layers, and finally a softmax classifier with 1000 class labels. Softmax layer is a normalizing layer which that takes as input a vector of $\mathrm{N}$ dimension, and converts it to a $\mathrm{N}$ probabilities vector which is proportional to the exponentials of the input elements (2), such as the values of the resulting vector are bounded in the interval $[0,1]$.

$\sigma(x)_{i}=\frac{e^{x_{i}}}{\sum_{j=1}^{N} e^{x_{j}}}$

With $\sigma: \mathbb{R}^{N} \rightarrow \mathbb{R}^{N}$ is the softmax function, $i=1, \ldots, N$ correspond to the index of the element in the $\mathrm{N}$ dimensional vector: $x=\left(x_{1}, \ldots, x_{N}\right)$. These different layers all aim at mitigating the vanishing gradient problem during backpropagation training. Indeed, convolutional layers produce feature maps based on the idea that an object could be found in different areas of the image, and pooling reduce the neighbouring features by taking feature map maximum.

Transfer Learning approach consisted basically of reusing the values of weights of the trained Deep Neural Network, while replacing the last layers with new ones which are retrained to provide a model that is better adapted to the target object and task on hand. Moreover, it required less time and calculation power. Indeed, the initialization weights are optimal and not random. Also, as the first layers in a Deep Learning Neural Network are extracting simpler features. These features and their corresponding weights remain similar provided that the model has been pre-trained on thousands of images. Indeed, Deep Learning has thousands of free parameters, which require thousands of images of training, in order to set these parameters to optimal values, which is a drawback as this Big Data model cannot be directly applied to train Small Data applications such as the one at our hands, where the quantity of annotated data is limited. Hence, the use of Transfer Learning is essential to retain the benefits of Deep Learning, while adapting to Small Data, as it needs to retrain only the last layers which correspond to higher level information that is important for the final decision.

The choice of using Transfer Learning was motivated by our dataset size that is relatively small, which is usually the case in specific satellite image and remote sensing applications, such as sand dunes detection, while retaining the predictive power of a Deep Learning model. Also, the processing power required by Deep Learning was enormous compared with Transfer Learning, which doesn't require as much iterations to converge. Thus using Transfer learning required less CPU cycles and less time.

\section{B. Proposed Method}

The proposed method started by an image pre-processing, followed by an image clustering, then Transfer Learning: 
1) Satellite image pre-processing: Satellite images are usually impacted by noise factors which could be optical or systemic. The use of a noise reduction strategy was essential. The proposed approch started by using a bilateral filter, represented by equation (3), which smooths the image while preserving edges, with a nonlinear combination of neighbouring pixel values.

$I^{\text {filtered }}(x)=\frac{1}{W_{p}} \sum_{x_{i} \in \Omega} I\left(x_{i}\right) f_{r}\left(\left\|I\left(x_{i}\right)-I(x)\right\|\right) g_{s}\left(\| x_{i}-\right.$

$x \|)$

with: $\left.W_{p}=\sum_{x_{i} \in \Omega} f_{r}\left(\left\|I\left(x_{i}\right)-I(x)\right\|\right) g_{s}\left(\left\|x_{i}-x\right\|\right)\right)$

$I$ was the original image and $I^{\text {filtered }}$ to the filtered image. $x$ corresponded to the coordinates of the pixel. $\Omega$ was the window centered in $x, x_{i} \in \Omega$ was a neighboring pixel, $f_{r}$ was a Gaussian function for smoothing differences in coordinates and $W_{p}$ was the weight assigned with the spatial kernel $g_{s}$. Then histogram equalization was used to enhance the contrast of the image, thus evenly distributing the intensities of pixels on the histogram. These operations were applied on both multispectral and panchromatic images. Indeed, high resolution satellite images have usually two components: multispectral images providing spectral resolution with medium spatial resolution and a panchromatic image with high spatial resolution. A fusion was operated between multispectral and panchromatic images with a bilinear interpolation to increase the spatial resolution of the multispectral images using the panchromatic image.

2) Image clustering: An unsupervisied approach was used for the segmentation of sand dunes, which consisted of two steps: the first one was the combination of multispectral bands of the satellite image into an RGB image, followed by a conversion to the CIELAB color space, which was defined by the International Commission on Illumination (CIR) with $\mathrm{L}$ being the luminosity layer, and $\mathrm{a}$ and $\mathrm{b}$ the chromaticity-layers encoding the color information. In the second step, a Lab image was taken as input and operated a clustering based on K-Means segmentation to distinguish between different geological aspects of the area of interest, including sand dunes. Indeed, multispectral bands in satellite images allow to get information about the nature of the terrain using its reflectance in different wavelenghts. Specifically, the distinguishment was made between different mineral compositions of sand from the satellite images.

3) Transfer learning: The proposed approach started by creating a database by annotating 1000 image patches of $228 \times 228 \times 3$ containing three classes: barchan dunes, dunes collisions, and bare field. This particular size was selected to fit our object of interest which was barchans and also was close to the input layer size of AlexNet Deep Neural Network which was $227 \times 227 \times 3$. Furthermore, the dataset was augmented by performing random flips along the y axis, and resized the images and scaled the patches. Our dataset was then split into $70 \%$ of images for learning and 30\% for testing.
Finally, the last three layers of AlexNet were replaced by a fully connected layer, a softmax layer, and a classification output layer.

4) Combining clustering with transfer learning: The segmentation labeled each pixel with a class corresponding to a specific geological feature, including sand. Clusternig results were used as a binary mask representing sand, which could be part of a barchan dune, dunes collision or even sand trail left by a moving dune. Hence, the mask was used in combination with the information resulting from Transfer Learning, which classified the image patches of the terrain in three categories: Barchan dunes, dunes collisions or bare field. The information from the clustering and Transfer Learning was fusioned as following: The Transfer Learning was operated only in areas which the segmentation results classified as containing sand. Besides, the results of the clustering were validated when they were consistant with the results found by Transfer Learning. Indeed, when the clustering indicated that the pixels in the image patch contained sand, but the Transfer Learning classified it as bare field, the image patch was declared as bare field, as sand in this case corresponded to sand trails left by moving dunes.

\section{EXPERIMENTAL RESULTS}

\section{A. Satellite Image Dataset}

The experiments were conducted on an IKONOS high resolution satellite image, which consisted of 5 bands (Table.1). The high resolution image was taken by the IKONOS satellite on July $23^{\text {rd }}$, 2003, in the South of Morocco, near the city of Tarfaya, in the Sahara Desert, with coordinates in between $27^{\circ} 26^{\prime} 8.6621^{\prime \prime} \mathrm{N}, 13^{\circ} 08^{\prime} 5.2628^{\prime \prime} \mathrm{W}$ and $27^{\circ} 41^{\prime} 1.0350^{\prime} \mathrm{N}, 13^{\circ} 22^{\prime} 0720^{\prime}$ 'W.

Two data flows were used: In the first one, the entire image was used to feed the clustering based image segmentation algorithm. In the second one, 1000 patches of $228 \times 228 \times 3$ were selected from the satellite image to be used for Transfer Learning after annotation. The dataset was split using a ratio of $70 \%$ for the training set $30 \%$ for the test set. The next section presents both quantitative and qualitative results.

\section{B. Image Pre-Processing}

This step aimed at enhancing each band image so as to improve subsequent processing. (Fig.2) showed the results after applying histogram equalization, then the bilateral filter. One can notice that the original image was very dark, with a poorly distinguishable objects of interest.

TABLE. I. SPECTRAL AND SPATIAL RESOlution of IKONOS IMAGE

\begin{tabular}{|l|l|l|l|l|}
\hline \multicolumn{2}{|c|}{} & Spatial resolution & Wavelength \\
\hline \multirow{3}{*}{ Bands } & Panchromatic & $0.82 \mathrm{~m} /$ pixel & $0.526-0.929 \mu \mathrm{m}$ \\
\cline { 2 - 5 } & \multirow{3}{*}{$\begin{array}{l}\text { Multi- } \\
\text { spectral }\end{array}$} & $\begin{array}{l}\text { Near } \\
\text { Infrared }\end{array}$ & $4 \mathrm{~m} /$ pixel & $0.757-0.853 \mu \mathrm{m}$ \\
\cline { 3 - 5 } & Red & $4 \mathrm{~m} /$ pixel & $0.632-0.698 \mu \mathrm{m}$ \\
\cline { 3 - 5 } & & Green & $4 \mathrm{~m} /$ pixel & $0.506-0.595 \mu \mathrm{m}$ \\
\cline { 3 - 5 } & Blue & $4 \mathrm{~m} /$ pixel & $0.445-0.516 \mu \mathrm{m}$ \\
\hline
\end{tabular}




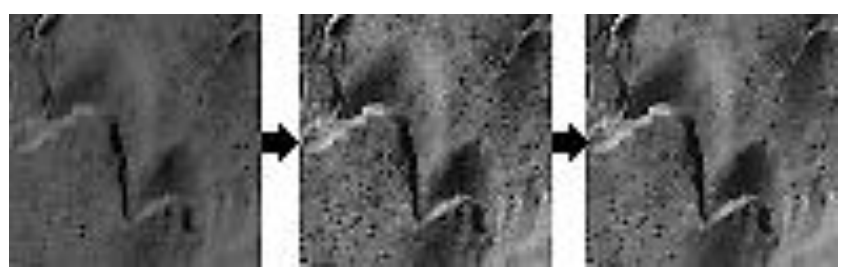

Fig. 2. Image Pre-Processing Results. from Left to Right: Raw Image Sample, Histogram Equalization, Bilateral Filter.

After enhacement, one could clearly observe the two dunes in the righmost image. Multispectral bands were also fusioned with the panchromatic band to improve the spatial resolution.

\section{Clustering-based Image Segmentation}

The proposed approach started by experimenting with different number of clusters $K=\{2,3,4,5,6\}$ in order to find the optimal number for our application. A set of 3 multispectral images was randomy selected, then converted to Lab color space, in order to isolate the chromatic information in the layers a and b. K-Means results can be observed in the first row of (Fig.3): For $\mathrm{K}=2$ and $\mathrm{K}=3$, classes were undersegmenting the image. Indeed, although, the central corridor of dunes was well segmented, however, the dunes it contained, which were considerd as foreground, were not distringuishable from their immediate surroundings, which were considered as a background in our application. For $\mathrm{K}=5$ and $\mathrm{K}=6$, classes were oversegmenting the satellite image as dunes, which were our object of interest, were split into several regions corresponding to different luminosities caused by the reflection of sun on their curved surfaces. Hence, the optimal number of clusters selected for the subsequent processing was $\mathrm{K}=4$, as it allowed to classify image regions into 4 categories corresponding to distinguishable geological items: The first 2 classes were considered as a background for our application, as they corresponded to solid bedrock. The third and fourth classes corresponded to sand from two different corridors. The main insterest of our application was the main dune corridor which contained the most important number of barchan dunes and was in the center of image. The secondary dune corridor which had a different mineral composition thus a different spectral response, was partially visible and felt mostly out of the limits of our satellite image borders. Therefore, the class which would be used as a mask was identified, then the process continued to find the best 3-channel combination of the 4 multispectral images, which provided the best segmentation results. For that purpose, the experimentation considered all 4 possibilities of multispectral bands 3-channel combinations: GNB, GRN, RGB, and NBR which were showed in the second row in (Fig.3). The subsequent evaluation was conducted by selecting a patch sample from the satellite image representing the most problematic area in the satellite image, as it contained barchan dunes, their collisions and bare field, including a road. A ground truth image of this evaluation patch depicting dunes was also used. The results of the statistical evalutaion of the 4 satellite images combinations were shown in (Table.2) which contains a confusion matrix and the following statistical indicators: accuracy, precision (or positive predictive value), F1-score, recall (or true positive rate), specificity (or true negative rate) and Youden's J-Statistic. The indicators showed the combination which demonstrated that the best segmentation was made using the RGB combination, having a $70 \%$ accuracy, $75 \%$ recall and a J-statistic of 0.44 .

\section{Transfer Learning}

AlexNet Deep Learning was used as a basis of the transfer learning. After annotating 1000 image patches extracted from the satellite image and labeled as barchan dunes, dunes collision or bare field, the dataset was augmented before launching the backpropagation algorithm with the training showed in (Fig.4). After 530 iterations on 10 epochs, using a minimum batch size of 10 images, and a validation frequency of 100 iterations, an accuracy of $84.01 \%$ was obtained. The training set and testing set examples were showed in (Fig.5).

TABLE. II. SEgMENTATION EVALUATION OF BANDS COMBINATIONS

\begin{tabular}{|c|c|c|c|c|c|c|c|c|c|}
\hline \multirow{4}{*}{\multicolumn{2}{|c|}{ Statistical Evaluation }} & \multicolumn{8}{|c|}{ Multispectral Band Combinations } \\
\hline & & \multicolumn{2}{|l|}{ GNB } & \multicolumn{2}{|l|}{ GRN } & \multicolumn{2}{|l|}{ NBR } & \multicolumn{2}{|l|}{ RGB } \\
\hline & & \multicolumn{2}{|c|}{ Actual class } & \multicolumn{2}{|c|}{ Actual class } & \multicolumn{2}{|c|}{ Actual class } & \multicolumn{2}{|c|}{ Actual class } \\
\hline & & sand & not sand & sand & not sand & sand & not sand & sand & not sand \\
\hline \multirow{2}{*}{ Predicted } & sand & 10081 & 22644 & 8159 & 28208 & 10270 & 20599 & 9970 & 16527 \\
\hline & not sand & 3157 & 30167 & 5079 & 24603 & 2968 & 32212 & 3268 & 36284 \\
\hline \multicolumn{2}{|l|}{ Total } & 13238 & 52811 & 13238 & 52811 & 13238 & 52811 & 13238 & 52811 \\
\hline \multicolumn{2}{|l|}{ Accuracy } & \multicolumn{2}{|l|}{0.6094} & \multicolumn{2}{|l|}{0.496} & \multicolumn{2}{|l|}{0.6432} & \multicolumn{2}{|l|}{0.7003} \\
\hline \multicolumn{2}{|c|}{ Precision (PPV) } & \multicolumn{2}{|l|}{0.3081} & \multicolumn{2}{|l|}{0.2244} & \multicolumn{2}{|l|}{0.3327} & \multicolumn{2}{|l|}{0.3763} \\
\hline \multicolumn{2}{|l|}{ F1-Score } & \multicolumn{2}{|l|}{0.4387} & \multicolumn{2}{|l|}{0.329} & \multicolumn{2}{|l|}{0.4657} & \multicolumn{2}{|l|}{0.5018} \\
\hline \multicolumn{2}{|c|}{ Recall (TPR) } & \multicolumn{2}{|l|}{0.7615} & \multicolumn{2}{|l|}{0.6163} & \multicolumn{2}{|l|}{0.7758} & \multicolumn{2}{|l|}{0.7531} \\
\hline \multicolumn{2}{|c|}{ Specificity (TNR) } & \multicolumn{2}{|l|}{0.5712} & \multicolumn{2}{|l|}{0.4659} & \multicolumn{2}{|l|}{0.6099} & \multicolumn{2}{|l|}{0.6871} \\
\hline \multicolumn{2}{|c|}{ Youden J-Statistic } & 0.3327 & & 0.0822 & & 0.3857 & & 0.4402 & \\
\hline
\end{tabular}




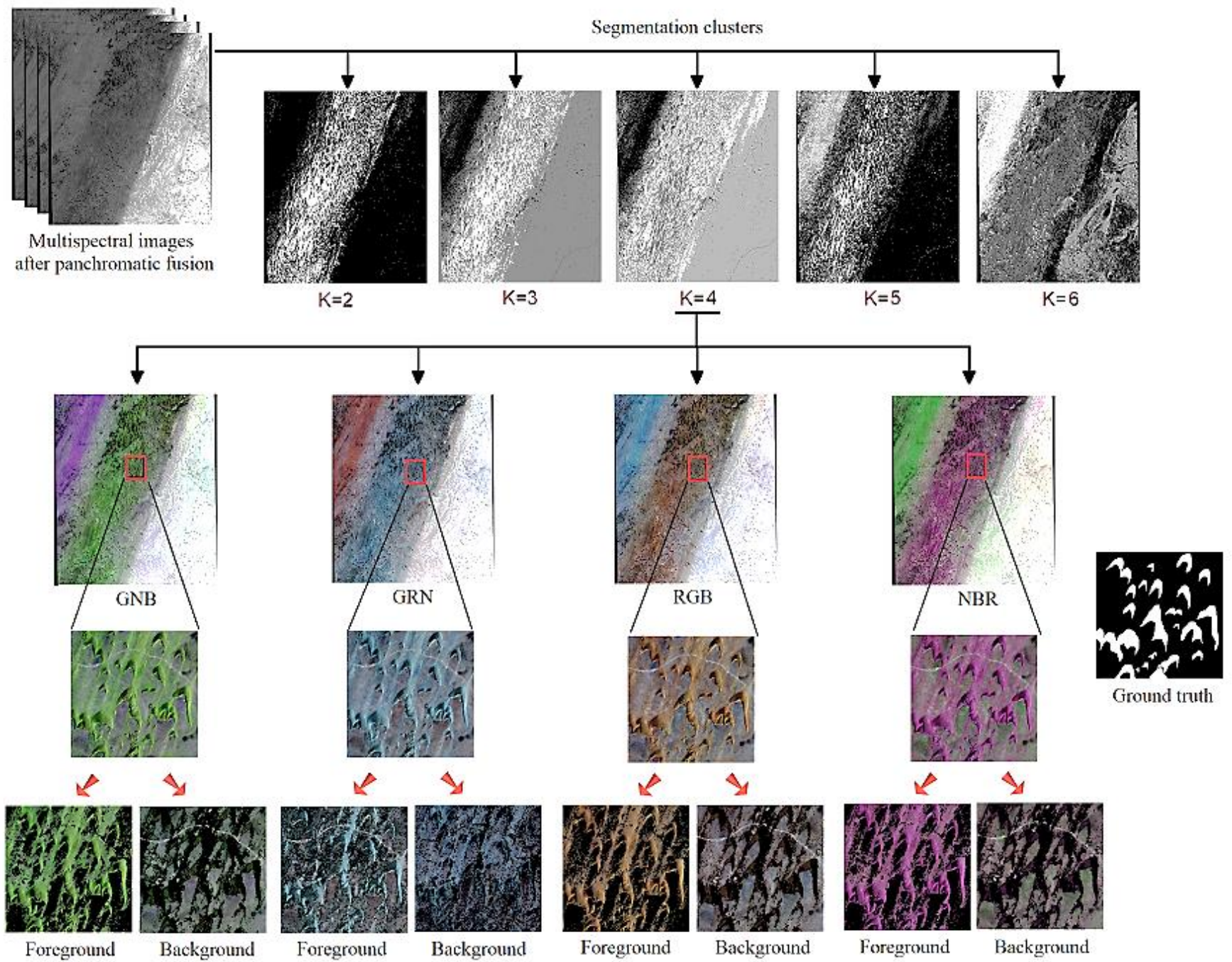

Fig. 3. Segmentation of Satellite Image Band Combinations, Evaluation Patches and Ground Truth.

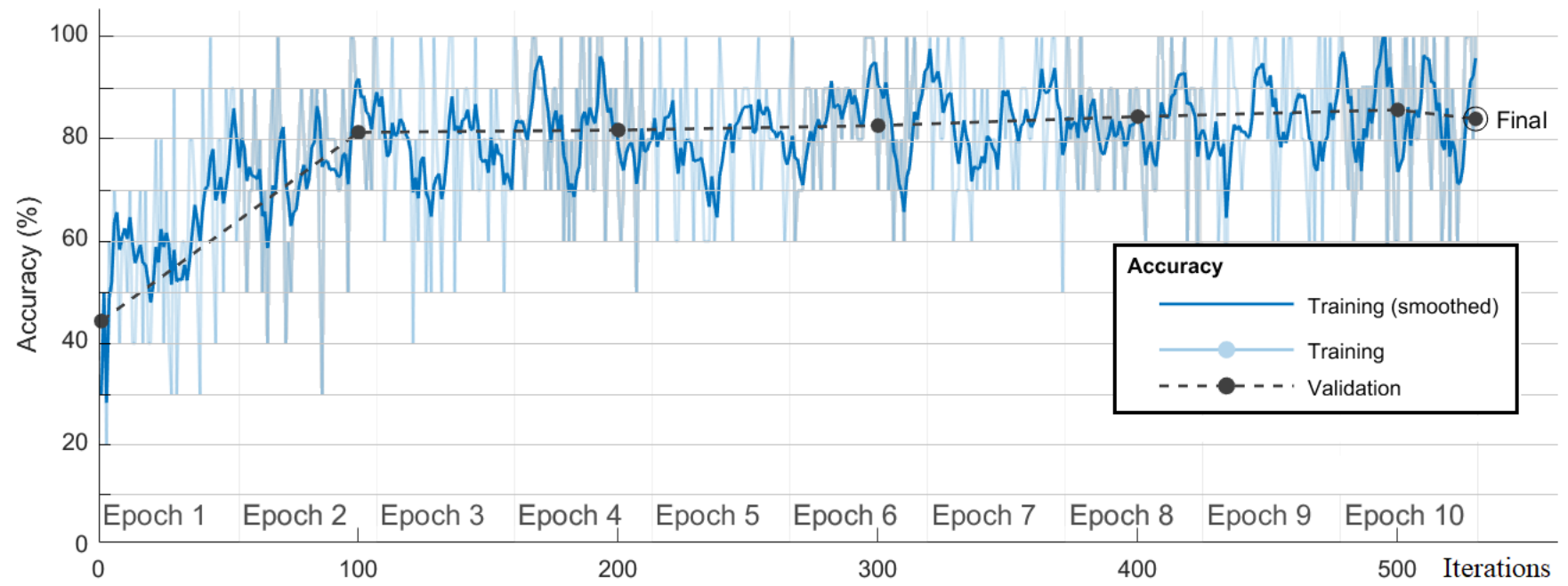

Fig. 4. Transfer Learning Training. 

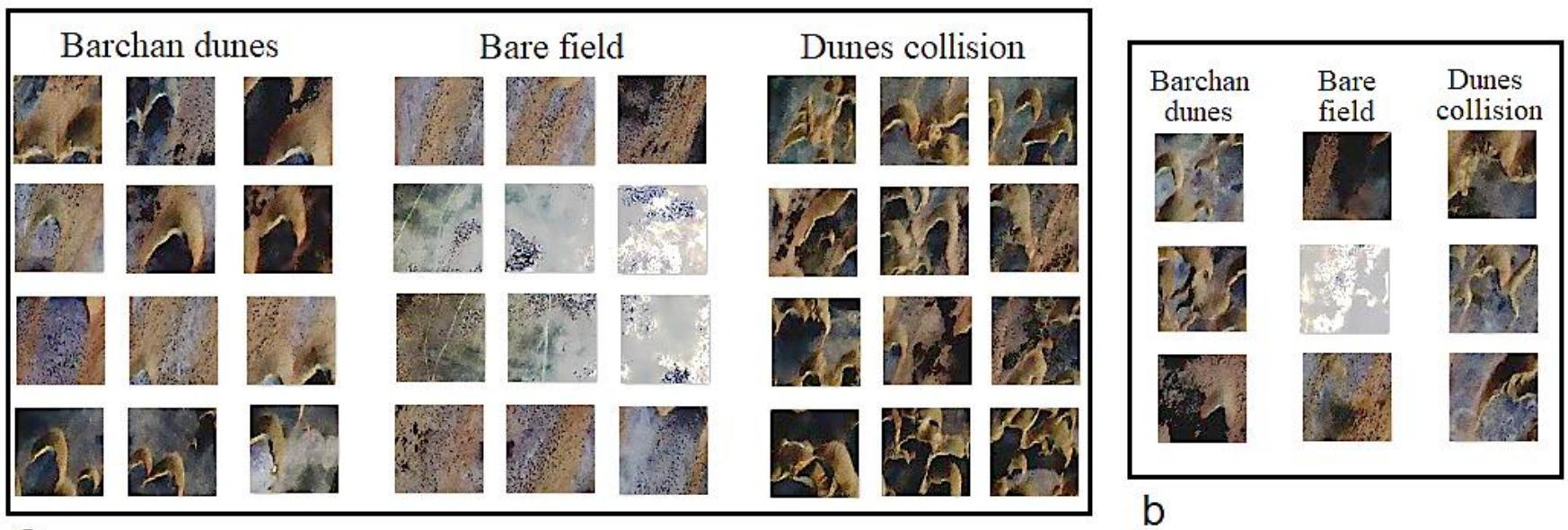

a

Fig. 5. Image Patches from the Satellite Image Representing 3 Classes of Objects (a): Training Set Examples, (b): Test Set Examples.

\section{DISCUSSION}

The dataset preparation benefited from data augmentation. During the first step, image enhancmened allowed data to be normalized but also enriched the multispectral bands after their fusion with the high resolution panchromatic band. On the second step, the primary segmentation eliminated the large surrounding areas containing the solid bedrock with an accuracy of virtually $100 \%$. the secondary segmentation results obtained (70\% accuracy) were also good considering the fact that they were evaluated against the patch showing the most problematic part of the satellite image, and also that the ground truth image used was approximative. Moreover, the secondary segmentation was targeting sand, while the ground truth depicted sand dunes only. Indeed, the evaluation patch at this stage, provided only with a reference to compare between the band combinations. The result was the selection of RGB combination as a basis for the third step. During this last step, one could notice that the misclassifications happened mainly between barchan dunes and dunes collisions classes as it was sometimes difficult to classify partially occluded barchan dunes. Conversely, the detections of bare field, including patches with sand trails were made seemlessly.

\section{CONCLUSION AND FUTURE WORK}

In this paper, a new method was proposed for the segmentation of barchans sand dunes and their collisions in high resolution satellite images. The contribution in this paper was the introduction of a new class consisting of barchans dunes collision, and the use of Transfer Learning as a variant of Deep Learning to achieve good results. Indeed, by combining a clustering method with Transfer Learning, an accuracy of $82.01 \%$ was obtained. This information is of interest to specialists studying sand dunes dynamics, which could come up with solutions which applicable at a large scale to protect the populations and the environment. In future work, the proposed method could benefit from the use of multiple high resolution satellite images, thus providing a multi temporal analysis of sand dunes progression. Also, the proposed approach could be improved in the future by taking into account the fact that Barchan sand dunes are 3 dimensional objects, which appeared as 2D crescent like objects in satellite images. A DSM (Digital Surface Model) could be generated from high resolution satellite images showing barchans dunes, their collisions and various desert features which could help understand the dynamics of desertification.

\section{REFERENCES}

[1] UNCCD: United Nations Convention to Combat Desertification in those countries experiencing serious drought and/or desertification, particularly in Africa. UNCCD explanatory leaflet, UNCCD Secretariat, Bonn 2008.

[2] Tsoar, H., 2001. Types of Aeolian Sand Dunes and Their Formation. Geomorphological Fluid Mechanics. N.J. Balmforth and A. Provenzale (Eds.): LNP 582, pp. 403-429.

[3] Elbelrhiti, H. and Hargitai H. Encyclopedia of Planetary Landforms, 2015, Chapter: Barchan, p123.

[4] M. A. Azzaoui, M. Adnani, H. El Belrhiti, I. E. Chaouki, L. Masmoudi . Segmentation of Crescent Sand Dunes in High Resolution Satellite Images using a Support Vector Machine for Allometry. International Journal of Advanced Computer Science and Applications. 2019. ISSN:2158-107X. Pages 191-198 Vol. 10, No. 11.

[5] McKee, E.D. Introduction to a study of global sand seas. McKee, E.D. (Ed.), A Study of Global Sand Seas: United StatesGeological Survey, 1979. 1052, pp. 3-19.

[6] Wolfe, S.A., Hugenholtz, C.H. Barchan dunes stabilized under recent climate warming on the northern Great Plains. 2009. Geology 37, 10391042.

[7] Kocurek, G. A., \& Ewing, R. C. Aeolian dune field self-organization Implications for the formation of simple versus complex dune-field $\begin{array}{llll}\text { patterns. Geomorphology. } 2005 . & 72(1-4), & 94-105 .\end{array}$ https://doi.org/10.1016/j.geomorph.2005.05.005

[8] Hugenholtz, C. H., and T. E. Barchyn. Spatial analysis of sand dunes with a new global topographic dataset: New approaches and opportunities, Earth Surf. Processes Landforms. 2010. 35, 986-992, doi:10.1002/esp.2013.

[9] Blumberg, D.G.. Remote sensing of desert dune forms b. polarimetric Synthetic Aperture Radar (SAR). Remote Sensing. of Environment 1998. 65, 204-216.

[10] Vermeesch, P., Drake, N., Remotely sensed dune celerity and sand flux measurements of the world's fastest barchans (Bodélé, Chad). Geophysical Research Letters. 2008. 35, L24404.

[11] Bishop, M.A.,. Comparative nearest neighbor analysis of megabarchanoid dunes, Ar Rub al Khali sand sea: the application of geographical indices to the understanding of dune field selforganization, maturity and environmental change. Geomorphology 2010. 120, 186194. 
[12] T. Blaschke, Object based image analysis for remote sensing, ISPRS Journal of Photogrammetry and Remote Sensing, Volume 65, Issue 1, 2010, Pages 2-16, ISSN 0924-2716, DOI: 10.1016/j.isprsjprs. 2009.06.004.

[13] Schmidhuber, J. Deep learning in neural networks: an overview. Neural Networks 2015. 61, 85-117.

[14] Litjens, G., Kooi, T., Bejnordi, B.E., Setio, A.A.A., Ciompi, F., Ghafoorian, M., Sanchez, C.I. A survey on deep learning in medical image analysis. Med. Image Anal. 2017. 42, 60-88.

[15] Ian J. Goodfellow, Jean Pouget-Abadie, Mehdi Mirza, Bing Xu, David Warde-Farley, Sherjil Ozair, Aaron Courville, Yoshua Bengio. Generative Adversarial Networks. 2014. arXiv:1406.2661.

[16] Simonyan, K., Zisserman, A. Very deep convolutional networks for large-scale image recognition. arXiv 2014. Available: https://arxiv.org/pdf/1409.1556.pdf.

[17] He, K., Zhang, X., Ren, S., Sun, J.. Deep residual learning for image recognition. In: IEEE Conference on Computer Vision and Pattern Recognition (CVPR) 2016, pp. 770-778.
[18] Szegedy, C., Ioffe, S., Vanhoucke, V., Alemi, A.A.. Inception-v4, inception-resnet and the impact of residual connections on learning. In: Proceedings of the Thirty-First AAAI Conference on Artificial Intelligence (AAAI-17). 2017, pp. 4278-4284.

[19] Chen, Y., Lin, Z., Zhao, X., Wang, G., Gu, Y. Deep learning-based classification of hyperspectral data. IEEE J. Sel. Top. Appl. Earth Obs. Remote Sens. 2014.7 (6), 2094-2107.

[20] Zhu, X.X., Tuia, D., Mou, L., Xia, G., Zhang, L., Xu, F., Fraundorfer, F. Deep learning in remote sensing: a comprehensive review and list of resources. IEEE Geosci. Remote Sens. Mag. 2017.5 (4), 8-36.

[21] Zhang, L., Zhang, L., Du, B. Deep learning for remote sensing data: a technical tutorial on the state of the art. IEEE Geosci. Remote Sens. Mag. 2016. 4 (2), 22-40.

[22] Krizhevsky, A., Sutskever, I., Hinton, G. Imagenet classification with deep convolutional neural networks. In: Advances in Neural Information Processing Systems. 2012. pp. 1097-1105. 